\title{
RESOLUÇÃO DO PLANO DE ESTIVA INTEGRADO À PROGRAMAÇÃO DE GUINDASTES PORTUÁRIOS VIA REPRESENTAÇÃO POR REGRAS
}

\author{
Anibal Tavares de Azevedo \\ UNICAMP \\ anibal.azevedo@fca.unicamp.br \\ Luiz Leduino de Salles Neto \\ UNIFESP \\ luiz.leduino@unifesp.br \\ Antônio Augusto Chaves \\ UNIFESP \\ antonio.chaves@unifesp.br \\ Antônio Carlos Moretti \\ UNIFESP \\ antonio.moretti@fca.unicamp.br
}

\begin{abstract}
RESUMO
A eficiência de um terminal portuário depende um planejamento apropriado da movimentação de contêineres, também chamado de plano de estiva. A partir do plano de estiva é possível estimar o tempo, e assim também o custo, para realizar os processos de carregamento e descarregamento de contêineres de um navio. Para tanto, este artigo propõe uma nova abordagem para resolver o problema de planejamento de estiva 3D integrado ao problema de programação ou scheduling de guindastes portuários. Ambos os problemas são NP-Hard e demanda grande número de variáveis binárias para representar uma solução em uma formulação inteira. A alternativa empregada neste trabalho é a aplicação sucessiva da representação por regras para o problema de estiva e o programação de guindastes portuários com um algoritmo genético. Soluções para problemas de grande porte que demandam aproxidamente 44 milhões de restrições e 42 milhões de variáveis são obtidas em menos de 15 minutos.
\end{abstract}

PALAVARAS-CHAVE: Plano de estiva, Programação de Guindastes Portuários, Representação por Regras, Algoritmo Genético. 


\section{Introdução}

De acordo com (Guan et al. 2013; Steenken et al. 2004), as operações em um terminal portuário de contêineres podem ser divididas em cinco problemas principais:

(1) Alocação de berços: a saída deste problema é a programação de qual navio será atendido em qual berço de modo que uma distância mínima de segurança seja respeitada entre dois navios, e dois navios não podem compartilhar o mesmo berço no mesmo período de tempo;

(2) Plano de estiva: este problema consiste em determinar como organizar os contêineres em um navio de modo a minimizar o número de movimentos de carregamento e descarregamento;

(3) Designação de Guindastes no Cais: o tempo que o navio porta-contêiner leva para carregar ou descarregar depende da programação de guindastes portuários alocados para cada seção do navio. Os guindastes não podem ultrassapar uns aos outros por terem seus movimentos limitados a um trilho comum. Além disso, uma distância de segurança entre eles também deve ser observada;

(4) Transporte do Cais: é necessário determinar que máquinas serão empregadas e quais serão suas trajetórias ligando o navio ao pátio do porto e vice-versa;

(5) Transporte do Pátio: A organização de pilhas no pátio do porto depende de como realizar operações eficientes para empilhar e desempilhar os contêineres que são trazidos ou levados por caminhões e trens.

Este trabalho irá abordar a resolução conjunta dos problemas (2) e (3). Para a resolução do problema (2) é necessário definir um plano de estiva que é fortemente relacionado com a estrutura especial do navio porta-contêiner como dado na Figura 1.
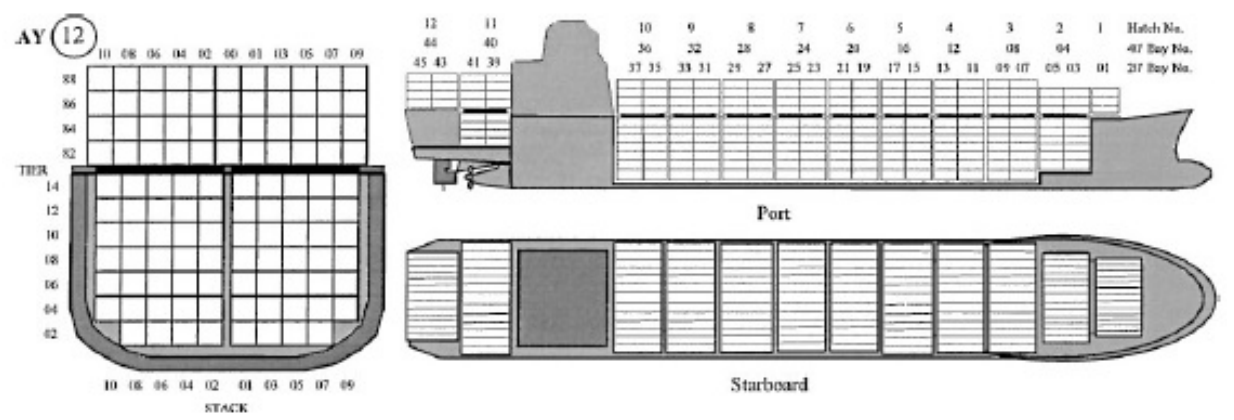

Figura 1: Estrutura de um navio porta-contêiner (Source: Wilson and Roach 2000).

O passo seguinte ao plano de estiva, é a definição da programação de guindastes portuários tal como dado na Figura 2. 


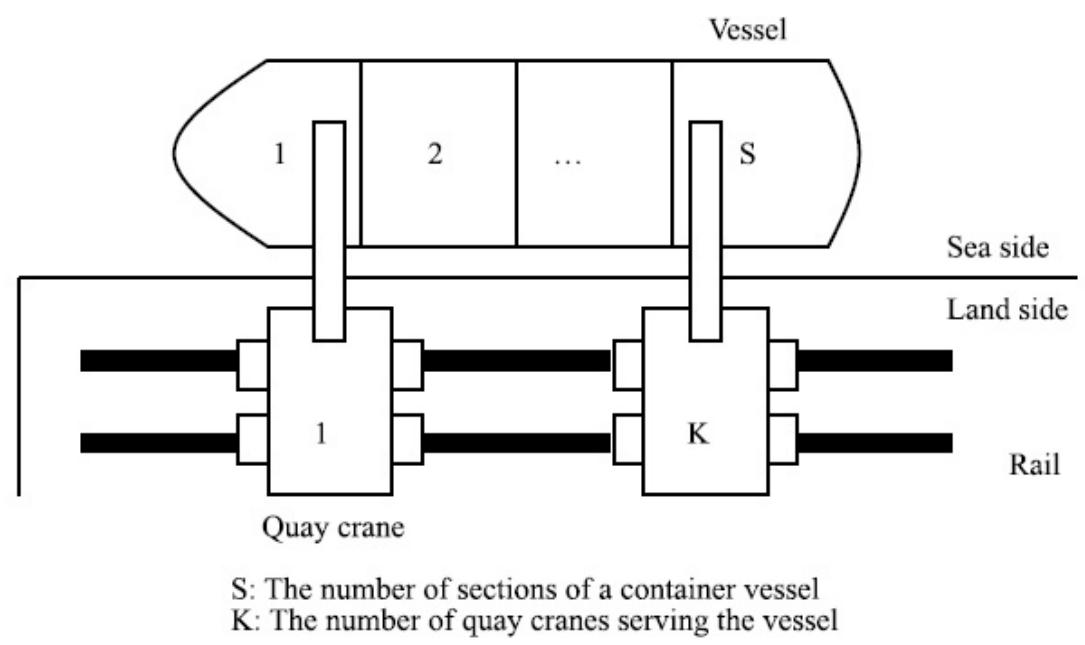

Figura 2: Problema de programação de guindastes portuários (Source: Lee et al. 2008).

Para evidenciar a complexidade de realizar a otimização conjunta de tais problemas, na literatura são encontradas ou abordagens que tratam do problema de estiva (Ambrosino et al., 2006 e 2010; Avriel et al., 1993, 1998; Botter e Brinati, 1991; Dubrovsky et al., 2002; Fan et al., 2010, Imai et al., 2006; Wilson e Roach, 2000) ou do problema de programação de guindastes portuários (Guan et al., 2013; Homayouni et al., 2011; Javanshir e Ganji, 2010; Lee et al, 2008; Legato et al., 2008; Mak e Sun, 2009). A otimização conjunta desses dois problemas é a contribuição original deste artigo.

\section{Modelo Matemático}

O mesmo modelo adotado para o plano de estiva em (Azevedo et al. 2012) será empregado neste artigo de modo que apenas a descrição do que as restrições representam será apresentada aqui. As seguintes considerações foram adotadas:

(a) O navio porta-contêiner possui um formato rectangular e pode ser representado por uma matriz com linhas $(r=1,2, \ldots, R)$, colunas $(c=1,2, \ldots, C)$ e baias $(d=1,2, \ldots, D)$ totalizando uma capacidade máxima de R x C x D contêineres;

(b) Todos os contêineres possuem o mesmo peso e tamanho;

(c) Todos os navios começam a ser carregados no porto 1 e sem nenhuma carga inicial;

(d) $\mathrm{O}$ navio visita os portos $2,3, \ldots, \mathrm{N}$ tal que o navio estará vazio no último porto, pois é considerado que o mesmo realiza uma rota circular onde o porto $\mathrm{N}$, na verdade, representa $\mathrm{o}$ porto 1;

(e) Em cada porto i = 1, 2, ..., N, o navio deve ser carregado com contêineres cujo destino são portos $\mathrm{i}+1, \ldots, \mathrm{N}$.

(f) O navio porta-contêineres pode sempre carregar todos os contêineres disponíveis em cada porto e isto não irá exceder sua capacidade máxima.

A formulação matemática correspondente às restrições (a)-(f) estão descritas no modelo matemático dado pelas Eqs (1)-(8). 


$$
f(x)=\square \square_{1}(x)+\square \square_{2}(y)
$$

Min

s.a:

$$
\begin{array}{ll}
\square_{v=i+1}^{j} \prod_{r=1}^{R} \prod_{c=1}^{c} \prod_{d=1}^{D} x_{i j v}(r, c, d) \square \prod_{k=1}^{i \square 1} \prod_{r=1}^{R} \square_{c=1}^{C} \prod_{d=1}^{D} x_{k j i}(r, c, d)=T_{i j} & i=1, \cdots, N \square 1, j=i+1, \cdots, N ; \\
\prod_{k=1}^{j} \prod_{j=i+1}^{N} \prod_{v=i+1}^{j} x_{k j v}(r, c, d)=y_{i}(r, c, d) & i=1, \cdots, N \square 1, r=1, \cdots, R ; \\
y_{i}(r, c, d) \square y_{i}(r+1, c, d) \square 0 & c=1, \cdots, C ; d=1, \cdots, D \\
& i=1, \cdots, N \square 1, r=1, \cdots, R \square 1 ; \\
\prod_{i=1}^{j ! 1} \prod_{p=j}^{N} x_{i p j}(r, c, d)+\prod_{i=1}^{j \square 1} \prod_{p=j+1}^{N} \prod_{v=j+1}^{p} x_{i p v}(r+1, c, d) \square 1 & i=2, \cdots, N, r=1, \cdots, R \square 1 ; \\
x_{i j v}(r, c, d)=0 \text { ou } 1 ; y_{i}(r, c, d)=0 \text { ou } 1 & c=1, \cdots, C ; d=1, \cdots, D \\
& i, j, v=1, \cdots, N, r=1, \cdots, R ; \\
& c=1, \cdots, C ; d=1, \cdots, D
\end{array}
$$

onde: a variável binária $x_{i j v}(r, c, d)$ é definida de forma que assume o valor 1 se existir um contêiner no compartimento $(r, c, d)$ que foi ocupado no porto i e tem como destino final o porto $\mathrm{j}$ e movido no porto $\mathrm{v}$; caso contrário assume valor zero. Por compartimento $(r, c, d)$ entende-se a linha $r$, a coluna $c$ e a baia $d$ no compartimento de carga do navio. É necessário salientar que a numeração das linhas é feita de baixo para cima, assim a linha de número 5 está acima da linha de número 4, a numeração das colunas é feita da esquerda para a direita e a profundidade é iniciada da popa até a proa. Similarmente, a variável $y_{i}(r, c, d)$ possui valor 1 se saindo do porto $i$ o compartimento $(r, c, d)$ for ocupado por um contêiner; caso contrário assume valor 0 .

A restrição (2) é a restrição de conservação de fluxo, onde $T_{i j}$ é o elemento da matriz de transporte que representa o número de contêineres que embarcam no porto i com destino ao porto j. A restrição (3) garante que cada segmento de rota tem pelo menos um único contêiner. A restrição (4) é necessária para garantir que existem contêineres embaixo do contêiner que ocupa o compartimento $(r, c)$. A restrição (5) é responsável por definir a movimentação dos contêineres: se um contêiner que ocupa a posição $(r, c, d)$ é descarregado no porto $j$, então, ou não existem contêineres acima dele, ou o índice $v$ do contêiner que ocupa o compartimento $(r+1, c, d)$ não é maior que j. A função objetivo da Eq. (1) possui é uma composição de dois diferentes critérios: o primeiro depende da movimentação dos contêineres, $\square_{h}(x)$, e o segundo depende posição ocupada pelos contêineres em cada porto, $\square_{2}(y)$. Os dois critérios podem ser combinados através de valores escalares fornecidos para os pesos $\square$ e $\square$ dentro de um modelo de otimização bi-objetivo. 
O termo $\square_{1}(x)$ relativo ao custo total de movimentação dos contêineres (assumindo que a movimentação de um contêiner possui um custo unitário e igual para todos os portos) em todos os portos é dado pela Eq. (7).

$$
\square_{1}(x)=\prod_{i=1}^{N \square 1} \prod_{j=i+1 v=i+1}^{N} \prod_{r=1}^{j \square 1} \prod_{c=1}^{R} \prod_{d=1}^{D} x_{i j, v}^{s}(r, c, d)
$$

O termo $\square_{2}(y)$ é relativo à estabilidade do navio de acordo com a posição dos contêineres (assumindo que cada contêiner possui um peso unitário e igual para todos os portos) em todos os portos no cenário $s$ e é dado pela Eq. (8).

$$
\square_{2}(y)=\bigsqcup_{i=1}^{N} \prod_{d=1}^{D}\left(\square x c m_{d i}^{s}\right)^{2}+\square_{d=1}^{D}\left(\square z c m_{d i}^{s}\right)^{2}
$$

$$
\begin{aligned}
& \text { onde: } \square z c m_{d i}^{s}=z_{c m}^{s} \square R / 2, \square x c m_{d i}^{s}=x c m_{d i}^{s} \square C / 2 \text { e } \\
& z c m_{d i}^{s}=\prod_{\square}^{R} \prod_{c=1}^{C}\left(y_{i}^{s}(r, c, d) \square(r \square 0.5)\right) \stackrel{\square}{\square} / \prod_{\square=1}^{R} \prod_{c=1}^{C} y_{i}^{s}(r, c, d) \stackrel{\square}{\square}
\end{aligned}
$$

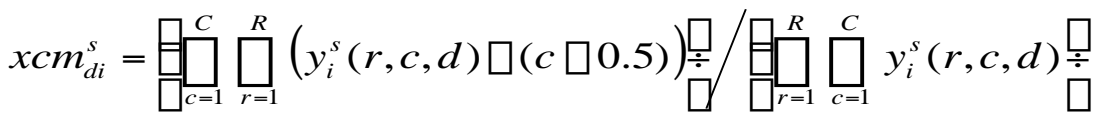

Observe na Eq. (8), que em cada cenário s para se calcular a soma dos desvios $\square x c m_{d i}^{s} \mathrm{e}$ $\square z c m_{d i}^{s}$ entre as coordenadas do centro de massa $\left(x \mathrm{~cm}_{d}^{s}, z_{\mathrm{c}} \mathrm{m}_{d}^{s}\right)$ e as coordenadas do centro geométrico $(R / 2, C / 2)$ de cada baia $d$, é necessário calcular as coordenadas do centro de massa $x c m_{d i}^{s}$ e $z c m_{d i}^{s}$, relativas respectivamente ao eixo das colunas $c$ e linhas $r$ de cada baia $d$.

Para a programação dos guindastes portuários o modelo descrito em (Lee et al. 2008) foi adotado e as seguintes considerações foram realizadas sob a forma de restrições e função objetivo:

(a) Ao invés de se considerar o navio dividido em seções como em (Lee et al. 2008), foi considerada cada baia do navio. Dessa forma, é possível transformar a informação de movimentação de contêineres fornecida pelo plano de estiva sob a forma do total de esforço necessário por baia;

(b) Foi assumido que o esforço total equivale ao número de contêineres que devem ser movidos e que cada container demanda uma unidade de tempo para ser carregado ou descarregado;

(c) Cada baia no navio só pode ter um único guindastes em um dado período de tempo; 
(d) Uma vez que um guindaste começou o serviço (carregamento ou descarregamento) em uma baia, o mesmo só irá parar quando não houver mais serviço para realizar;

(e) Quando um guindaste troca sua operação de uma baia para outra, ele leva um tempo constante e igual a três unidades de tempo para mover um contêiner;

(f) Uma distância minima entre os guindastes deve ser observada. Isto significa que, às vezes, um guindaste não pode ser movido e não pode começar o seu serviço até que um segundo guindaste termine o seu trabalho e mova-se para uma nova posição que garanta a distância mínima entre os guindastes;

(g) Um guindaste não deve ultrapassar outro, pois eles compartilham o mesmo trilho;

(h) Todos os guindastes possuem a mesma e constant taxa de serviço de movimentação de uma unidade de trabalho por unidade de tempo.

O modelo matemático em termos de programação linear com variáveis binárias para o problema de programação de Guindastes Portuários proposto por (Lee et al. 2008) é dado pelas Eqs. (9)-(21).

$$
\begin{aligned}
& \text { Min } \square \\
& t x_{j t} \square \square \text {, } \\
& \mathrm{j}=1, \ldots, \mathrm{S} ; \mathrm{t}=1, \ldots, \mathrm{T} \\
& w_{j t} \square M x_{j t} \square 0 \text {, } \\
& \mathrm{j}=1, \ldots, \mathrm{S} ; \mathrm{t}=1, \ldots, \mathrm{T} \\
& w_{j t \square 1} \square \measuredangle \nabla_{j, j}^{t \square 1, t} \square w_{j t}, \\
& \mathrm{j}=1, \ldots, \mathrm{S} ; \mathrm{t}=2, \ldots, \mathrm{T} \\
& z_{j, j+1}^{t, t+1}+z_{j, j \square 1}^{t, t+1}+z_{j, j}^{t, t+1}=b_{j}, \\
& \mathrm{j}=1, \ldots, \mathrm{S} ; \mathrm{t}=1 \\
& z_{j \square 1, j}^{t \square 1, t}+z_{j, j}^{t \square 1, t}+z_{j+1, j}^{t \square 1, t} \square z_{j, j+1}^{t, t+1} \square z_{j, j \square 1}^{t, t+1} \square z_{j, j}^{t, t+1}=0, \\
& \mathrm{j}=1, \ldots, \mathrm{S} ; \mathrm{t}=2, \ldots, \mathrm{T}-1 \\
& Z_{j \square 1, j}^{t \llbracket 1, t}+Z_{j, j}^{t \square 1, t}+Z_{j+1, j}^{t \llbracket 1, t}=Z_{j, j+1}^{t, t+1} \\
& \mathrm{j}=1, \ldots, \mathrm{S} ; \mathrm{t}=\mathrm{T} \\
& \prod_{j=1}^{S} z_{j, 1}^{t \square 1, t}=\prod_{j=1}^{S} b_{j}, \\
& \mathrm{t}=\mathrm{T}+1 \\
& z_{j \square 1, j}^{t \square 1, t}+z_{j, j}^{t \square 1, t}+z_{j+1, j}^{t \square 1, t} \square 1 \\
& j=1, \ldots, S ; t=2, \ldots, T \\
& z_{j \square 1, j}^{t \square 1, t}+z_{j, j}^{t \square 1, t} \square 1 \\
& j=1, \ldots, S ; t=2, \ldots, T \\
& y_{j, j}^{t \square 1, t} \square z_{j, j}^{t \square 1, t} \square 0 \\
& j=1, \ldots, S ; t=2, \ldots, T \\
& y_{j, j}^{t \square 1, t}+x_{j t} \square y_{j, j}^{t, t+1} \square 1 \\
& j=1, \ldots, S ; t=2, \ldots, T-1 \\
& w_{j t} \square 0 \text { with } w_{j 1}=W_{j}, x_{j t}, y_{j, j}^{t, t+1}, z_{j, j, '}^{t, t+1} \square[0,1] \quad \mathrm{j}, \mathrm{j}^{\prime}=1, \ldots, \mathrm{S} ; \mathrm{t}=1, \ldots, \mathrm{T}-1
\end{aligned}
$$

Onde: $\mathrm{M}$ é um valor grande, $\mathrm{t}$ é o índice relativo aos períodos de tempo, $\mathrm{T}$ é o horizonte de tempo que pode ser qualquer valor maior que o Makespan $\square \square$ é uma ponderação acerca da carga de trabalho em uma dada seção $\mathrm{j}$ no período $t, b_{j}=1$ se um guindaste está originalmente localizado na seção j e 0 caso contrário, $W_{j}$ é a carga inicial de trabalho na seção j.

Para o modelo dado pelas Eqs (9)-(21) as seguintes variáveis de decisão foram consideradas:

(i) Uma variável binária $x_{j t}$ tem valor 1 , se ainda existir carga de trabalho na baia j no início do período t e 0 , caso contrário;

(ii) A variável inteira $w_{j t}$ representa a carga de trabalho remanescente na baia j no início do período de tempo t; 
(iii) A variável inteira $z_{j, j^{\prime}}^{t, t+1}$ representa o número de guindastes que se movem da baia j para a baia j' durante o período de tempo t;

(iv) A variável inteira $y_{j, j}^{t, t+1}$ representa o número de guindastes na baia j durante o período de tempo t.

A função objetivo consiste na minimização do tempo necessário para terminar os serviços no navio, incluindo movimentos de carregamento e descarregamento, e mudança de posição do guindaste. Assim, a função objetivo é a minimização do makespan. A restrição (10) determina que o tempo de término da última unidade de trabalho, i.e., o makespan. A restrição (11) estabelece a existência ou não da carga de trabalho restante na seção j no tempo t. A restrição (12) representa o balanceamento do fluxo de carga de trabalho da seguinte forma: para cada seção j existe uma carga inicial de trabalho $w_{j 1}=W_{j}$. Para cada período de tempo escolhido, a carga de trabalho remanescente será reduzida em unidades $\square$ antes de atingir zero, se houver um guindaste portuário operando durante o período de tempo selecionado. As restrições (13) até (16) são relativas a conservação do fluxo de guindastes. A restrição (17) indica que para cada unidade de tempo, cada seção pode comportar no máximo um único guindaste. A restrição (18) indica que um guindaste não pode cruzar outro. A restrição (19) indica que o guindaste que um guindaste pode permanecer inativo em uma seção j durante um período t. Finalmente, a restrição (20) representa restrições de não-preempção, isto é, um guindaste não pode começar uma nova tarefa até que a tarefa atual para qual foi alocado esteja terminada.

Deve ser destacado que os modelos matemáticos são apresentados para ilustrar a complexidade e o modo com os dois problemas podem ser acoplados. Neste sentido, pela primeira vez na literatura, os dois modelos descritos anteriormente foram acoplados ao se utilizar baias ao invés de seções no problema dos guindastes. Dessa forma, foi possível empregar a observação de que o resultado do problema de estiva ( $\mathrm{P}$ ), o número de movimentos por baia, pode ser transformado na entrada do problema de operação de guindastes $(\mathrm{O})$, o esforço total por baia, tal como dado na Fig. 3.

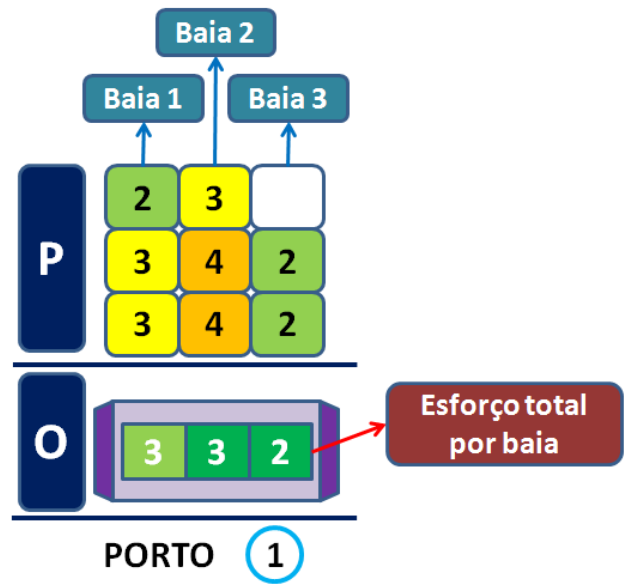

Figura 3: Acoplando o plano de estiva (P) com a operação de guindastes $(\mathrm{O})$.

É importante observar que ao realizar o acoplamento entre o plano de estiva (P) e a operação de guindastes (O), a resolução do problema (P) dependerá, em cada porto, da resolução do problema de guindastes (O). Assim, uma instância que considere um problema conjunto de (P) e (O) com um horizonte de 30 portos deverá resolver não só o problema (P) dado pelas Eqs. (1)(8), mas, também, 60 sub-problemas $(\mathrm{O})$ dado pelas Eqs. (9)-(21). Isto se deve ao fato de que, no problema conjunto, as operações de carregamento e descarregamento em cada porto devem ser realizadas através da programação e operação de guindastes portuários. 


\section{Método de Solução}

Como demonstrado em (Avriel e Penn 2000), o modelo matemático com variáveis binárias para o problema de estiva (P) não é adequado para problemas de grande porte. Caso a representação por variáveis binárias seja empregada, mesmo com a adoção de meta-heurísticas, o número de variáveis a serem empregadas cresce de forma proibitiva. No artigo (Azevedo et al. 2012, 2014), ao invés de empregar uma meta-heurística que usa variáveis binárias, é empregada uma representação alternativa na resolução do problema de estiva $(\mathrm{P})$ através integrada dos dois problemas através da representação por regras. Neste artigo, além de empregar a representação por regras, pela primeira vez, também para o problema de operação de guindastes (O), estas foram combinadas às regras do problema de estiva (P) para permitir a resolução integrada de ambos os problemas. A combinação entre as regras para carregamento e descarregamento de navios descritas em (Azevedo et al. 2012, 2014) com quatro regras de operações de guindastes tal como dado na Tabela 1.

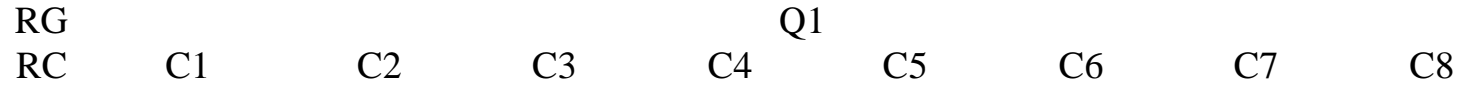

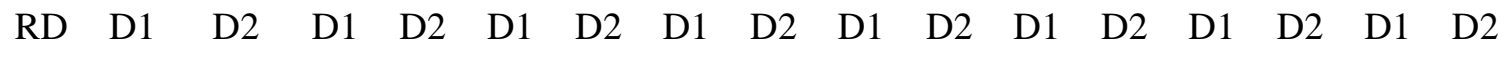

$$
\begin{aligned}
& \begin{array}{lllllllllllllllll}
\mathrm{R} & 1 & 2 & 3 & 4 & 5 & 6 & 7 & 8 & 9 & 10 & 11 & 12 & 13 & 14 & 15 & 16
\end{array}
\end{aligned}
$$

Na Tabela 1, RG representam as regras de alocação de guindastes, RC as regras de carregamento, $\mathrm{RD}$ as regras de descarregamento e $\mathrm{R}$ as regras finais que são combinações das demais. A Tabela 1 mostra apenas as primeiras 16 combinações das 64 possíveis de regras finais, pois foram utilizadas 4 regras de programação de guindastes.

A Fig. 4 ilustra o passo-a-passo do funcionamento de uma das regras de operação de dois guindastes portuários.

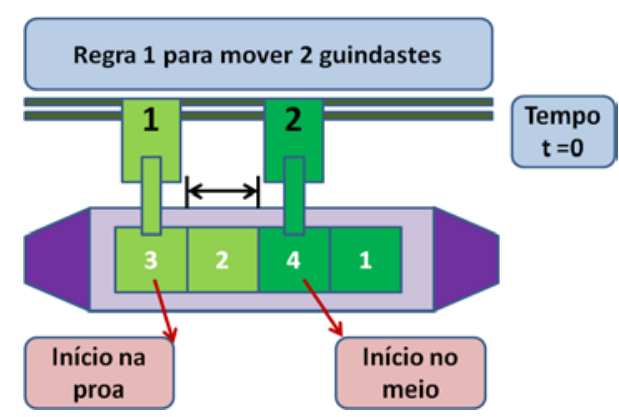

(A) Programação inicial de dois guindastes.

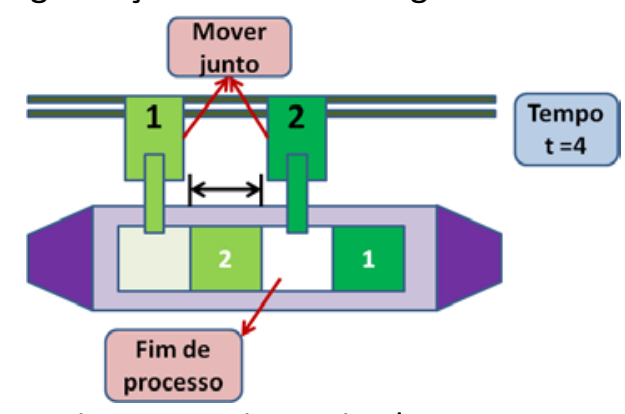

(C) Movimento sincronizada entre os dois guindastes.

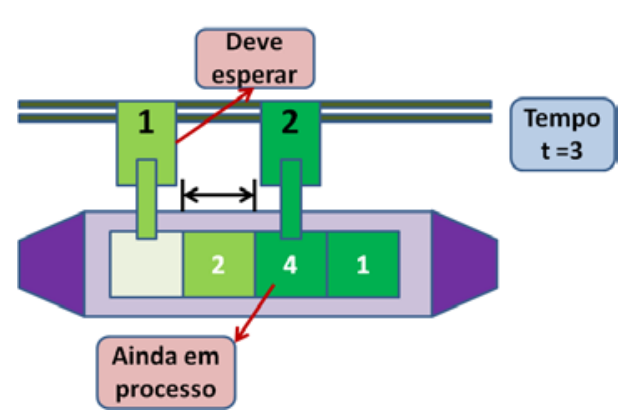

(B) Manter distância mínima de segurança.

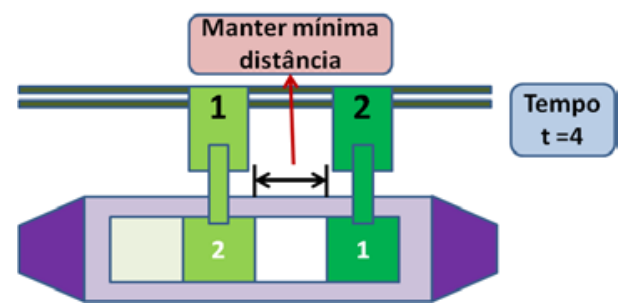

(D)A regra de movimentação é tal que respeita a restrição de distância mínima de segurança. 


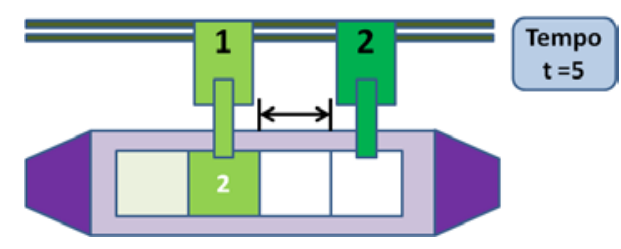

(E) Fim de serviço no guindaste 2, mas não no 1.

Figura 4: Descrição detalhada do funcionamento portuários.

Apesar do uso da representação por regras reduzir consideravelmente a complexidade do problema a ser resolvido, a decisão de qual regra, das que estão descritas naTabela 1 , deve ser aplicada em cada porto resulta em um problema combinatório. Para um problema que considera um horizonte de 30 portos, existem $64^{30}$, ou seja, aproximadamente $1,54 \times 10^{54}$, possíveis soluções. Assim, emprega-se um algoritmo genético para resolver o problema combinatório resultante.

A estrutura do algoritmo genético empregado neste trabalho é similar a descrita em (Azevedo et al., 2014), com a única diferença em relação à codificação que mapeia as soluções e os indivíduos da população.

Uma representação compacta da solução pode ser realizada através de um vetor $S$ cujo elemento $s[j]$ de valor igual a $k$ indica qual regra (daquelas fornecidas na Tabela 1 ) foi utilizada para o porto $j$ tal como dado na Figura 3. Então, no exemplo da Figura 5, tem-se que no porto 1, foi utilizada a regra 4 , no porto 2 a regra 3 e assim sucessivamente.

$$
\begin{array}{l|l|}
\cline { 2 - 2 } \text { P1 } & 4 \\
\text { P2 } & 3 \\
\text { P3 } & 2 \\
\hline
\end{array}
$$

Figura 5: Codificação do algoritmo genético para relacionar indivíduos e soluções.

A diferença entre a codificação apresentada em (Azevedo et al., 2014) e a deste artigo é que agora as regras não só definem a disposição dos contêineres no navio, mas, também, como deverá ser realizada a operação dos guindastes portuários, tal como ilustrado na Figura 4.

\section{Resultados Computacionais}

Foram obtidos resultados para instância com até cerca de 44 milhões de restrições e 42 milhões de variáveis binárias utilizando um programa criado em Matlab 7.0, e uma máquina com um processador $1.66 \mathrm{GHz}$ Core Duo Intel Processor, memória RAM memory de 2 GB e sistema operacional Windows Vista Operational System com Service Pack 2.Duas funções objetivos foram testadas: uma que busca minimizar o tempo total de operação (Fig. 6) e outra que busca minimizar a instabilidade do arranjo dos contêineres no navio (Fig. 7) para uma instância que considera a movimentação de cargas para um número de portos igual a 30 . Sem perda de generalidade foi suposto que o tempo de deslocamento do guindaste de uma baia para outra e o tempo de movimentação de uma carga seja para carga ou descarga no navio são iguais. Também foi suposto que os pesos e as dimensões de todos os contêineres são iguais. Além disso, tanto o problema de estiva (Fig. 6(A), (C); Fig. 7(A), (C)) quanto o problema de alocação de guindastes (Fig. 6(B), (D); Fig. 7(B), (D)) foram resolvidos de forma integrada, isto é, a resolução do problema de estiva (P) observa o impacto da aplicação das regras em termos do problema de alocação de guindastes (O). As soluções mostradas nas Figuras 6 e 7 correspondem aos melhores valores de função objetivo obtidos para a minimização do número de movimentos e a minimização da instabilidade das baias dos navios, respectivamente. 


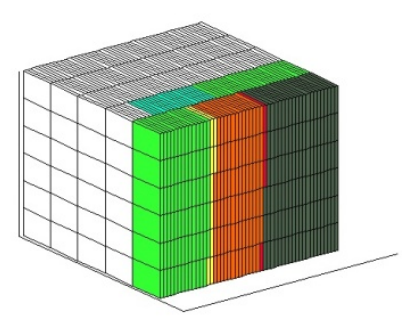

(A) Minimizando movimentos.

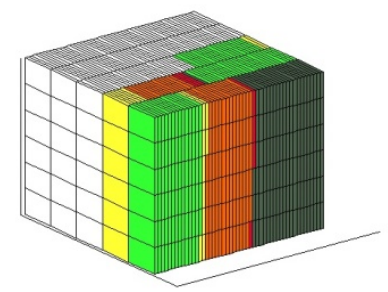

(C) Minimizando movimentos.

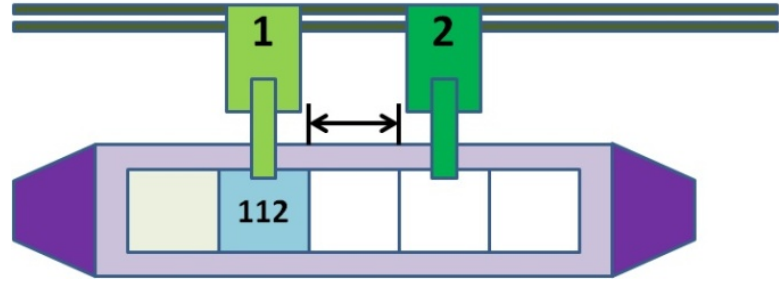

(B) Operação resultante de (A) no porto 2.

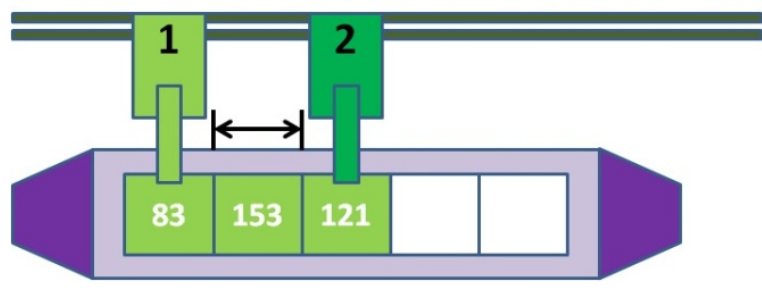

(D) Operação resultante de (C) no porto 3.

Figura 6: Solução em termos plano de estiva e operação de guindastes para $(\square \square=(1,0)$ na Eq. (1) de modo a minimizar o número de movimentos.

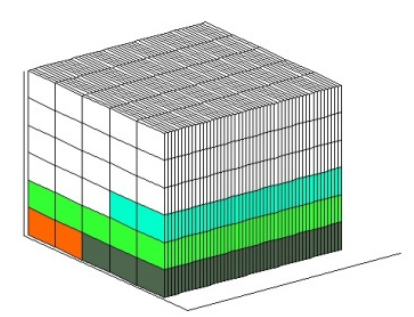

(A) Minimizando instabilidade.

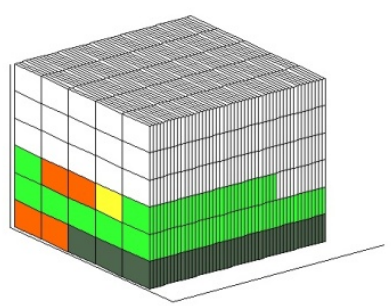

(C) Minimizando instabilidade.

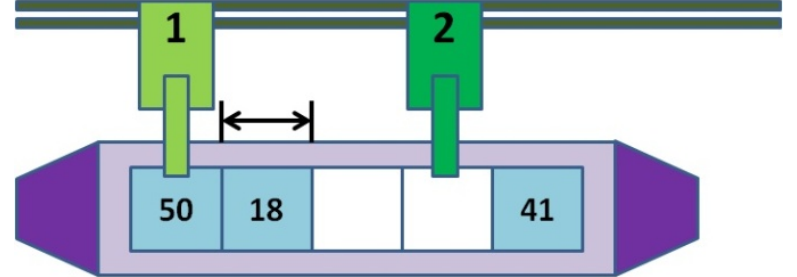

(B) Operação resultante de (C) no porto.

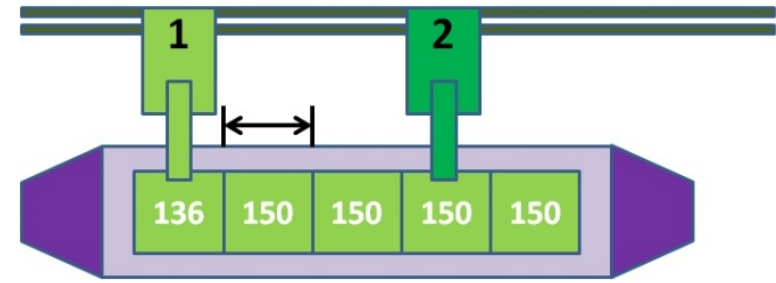

(D) Operação resultante de (C) no porto.

Figura 7: Solução em termos plano de estiva e operação de guindastes para $(\square \square=(0,1)$ na Eq. (1) de modo a minimizar a instabilidade.

Para o problema com maior número de portos, isto é trinta, foi possível resolver o problema de modo a obter soluções factíveis, bem como melhorar sua qualidade, em menos de 15 minutos, mesmo que o problema resultante tenha dimensão de 44 milhões de restrições e 42 milhões de variáveis apenas para o problema de estiva. 


\section{Conclusões e perspectivas futuras}

Neste trabalho, foi apresentado pela primeira vez, a resolução conjunta do problema de estiva e de alocação de guindastes, isto é, determinar o arranjo dos contêineres em um navio, mas de modo a observar o correspondente impacto em termos de tempo de operação dos guindastes. Para tanto, a representação por regras foi extendida para ser aplicada não só no problema do plano de estiva, mas também para determinar a programação de guindastes em um terminal portuário de contêineres. A metodologia proposta conseguiu obter soluções para problemas cujo modelo matemático correspondente possui cerca de 44 milhões de restrições e 42 milhões de variáveis em menos de 15 minutos. Diante da velocidade computacional para obter soluções factíveis e de boa qualidade para problemas complexos integrados, uma perspectiva futura é ampliar a utilização da representação por regras de modo a incluir outras operações portuárias tais como a movimentação de contêineres no pátio do porto, bem como considerar diferentes tipos de contêineres em termos de pesos, dimensões e conteúdo (carga tóxica, refrigerada, dentre outros).

Agradecimentos à FAPESP pelo apoio financeiro através dos processos 2010/51274-5 e 2014/03258-1.

\section{Referências}

Ambrosino, D., Schiomachen, A., Tanfani, E., (2006). A decomposition heuristics for the container ship stowage problem”, Journal of Heuristics 12, pp. 211-233.

Ambrosino, D., Anghinolfi, D., Paolucci, M., Sciomachen, A. (2010). An Experimental Comparison of Different Heuristics for the Master Bay Plan Problem, Lecture Notes in Computer Science 6049, pp. 314-325.

Avriel, M., Penn, M., (1993). Container ship stowage problem, Computers and Industrial Engineering, 5, pp. 271-274.

Avriel, M., Penn, M., Shpirer, N., Wittenboon, S. (1998). Stowage planning for container ships to reduce the number of shifts, Annals of Operations Research 76, pp. 55-71.

Avriel, M., Penn, M., Shpirer, N. (2000). Container ship stowage problem: complexity and connection to the coloring of circle graphs, Discrete Applied Mathematics 103, pp. 271-279.

Azevedo, A., Ribeiro, C., Sena, G., Chaves, A., Salles, L., Moretti, A. (2012). Solving the 3D Container Ship Loading Planning Problem by Representation by Rules and Beam Search, in ICORES'12, pp. 132-141.

Azevedo, A., Ribeiro, C., Sena, G., Chaves, A., Salles, L., Moretti, A. (2014). Solving the 3D Container Ship Loading Planning Problem by Representation by Rules and Meta-heuristics, International Journal of Data Analysis Techniques and Strategies - Special issue on: 'Optimization and Simulation Real-life Scenarios', Vol. 6, no. 3, pp. 228-260.

Botter, R., Brinati, M., (1991). Stowage Container Planning: a model for get-ting optimal solution. In: International Conference of Computer Applications in the Automation of Shipyard Operation and Ship Design, Rio de Janeiro, pp. 217-229.

Dubrovsky, O.; Levitin, G., Penn, M. (2002). A Genetic Algorithm with a Compact Solution Encoding for the Containership Stowage Problem, Journal of Heuristics 8, pp. 585-599.

Fan, L., Low, M., Ying, H., Jing, H., Min, Z., Aye, W. (2010). Stowage Planning of Large Containership with tradeoff between Crane Workload Balance and Ship Stability, Proceedings of the International MultiConference of Engineers and Computers Scientists III, pp. 1-7.

Guan, Y., Yang, K-H., Zhou, Z. (2013). The Crane Scheduling: models and solution approaches”, Annals of Operations Research 203, p. 119-139. 
Homayouni, S., Tang, S., Ismail, N., and Ariffin, M. (2011). Using simulated annealing algorithm for optimization of quay cranes and automated guided vehicles scheduling, International Journal of the Physical Sciences 6(27), pp. 6286-6294.

Imai, A., Sasaki, K., Nishimura, E., Papadimitriou, S. (2006) Multi-objetive simultaneous stowage and loading planning for a container ship with container rehandle in yard stacks, European Journal of Operational Research 171, pp. 373-389.

Javanshir, H., Ganji, S. (2010) Yard crane scheduling in port container terminals using genetic algorithm, J. Ind. Eng. Int. 6 (11), pp. 39-50.

Lee, D., Wang, D., Miao, L. (2008). Quay crane scheduling with non-interference constraints in port container terminals, Transportation Research E 44, pp. 124-135.

Legato, P., Mazza, R., Trunfio, R. (2008). Simulation-Based Optimization for the Quay Crane Scheduling Problem, WSC '08 Proceedings of the 40th Conference on Winter Simulation, pp. 2717-2725.

Mak, K., Sun, D. (2009). Scheduling Yard Cranes in a Container Terminal Using a New Genetic Approach, Engineering Letters 17(4), pp.274-280.

Steenken, D., Voss, S., Stahlbock, R. (2004). Container terminal operation and operations research - a classification and literature review, OR Spectrum 26 (1), pp. 3-49.

Wilson, I.,. Roach, P. (2000). Container stowage planning: a methodology for generating computerised solutions, Journal of the Operational Research Society 51, pp. 1248-1255. 